\title{
Spontaneous retroclival hematoma: a case series
}

\author{
Jared Narvid, MD, ${ }^{1}$ Matthew R. Amans, MD, ${ }^{2}$ Daniel L. Cooke, MD, ${ }^{2}$ Steven W. Hetts, MD, ${ }^{2}$ \\ William P. Dillon, MD, ${ }^{1}$ Randall T. Higashida, MD, ${ }^{2}$ Christopher F. Dowd, MD, ${ }^{2}$ and \\ Van V. Halbach, MD²
}

Divisions of ${ }^{1}$ Neuroradiology and ${ }^{2}$ Interventional Neuroradiology, Department of Radiology and Biomedical Imaging, University of California, San Francisco, California

OBJECTIVE Retroclival hematomas are rare, appearing mostly as posttraumatic phenomena in children. Spontaneous retroclival hematoma (SRH) in the absence of trauma also has few descriptions in the literature. None of the reported clinical cases features the combination of an SRH and intraventricular hemorrhage (IVH). Nevertheless, despite extensive cases of idiopathic or angiographically negative subarachnoid hemorrhage (SAH) of the posterior fossa, only a single case report of a patient with a unique spontaneous retroclival hematoma has been identified. In this study, the authors reviewed the presentation, management, and clinical outcome of this rare entity.

METHODS The authors performed a retrospective analysis of all patients with diagnosed SRH at their institution over a 3-year period. Collected data included clinical history, laboratory results, treatment, and review of all imaging studies performed.

RESULTS Four patients had SRH. All were appropriately evaluated for coagulopathic and/or traumatic etiologies of hemorrhage, though no etiology could be found. Moreover, all of the patients demonstrated SRH that both clearly crossed the basioccipital synchondrosis and was contained within a nondependent configuration along the retroclival dura mater.

CONCLUSIONS Spontaneous retroclival hematoma, often associated with IVH, is a rare subtype of intracranial hemorrhage frequently recognized only when MRI demonstrates compartmentalization of the posterior fossa hemorrhage. When angiography fails to reveal an underlying lesion, SRH patients, like patients with traditional angiographically negative $\mathrm{SAH}$, enjoy a remarkably good prognosis.

http://thejns.org/doi/abs/10.3171/2015.2.JNS142221

KEY WORDS clivus; intracranial hematoma; subarachnoid hemorrhage; vascular disorders

$\mathrm{R}$ ETROCLIVAL hematomas are rare and appear mostly as posttraumatic phenomena in children..$^{10}$ Spontaneous retroclival hematoma (SRH) in the absence of trauma also has few descriptions in the literature. ${ }^{8,9,11}$ None of the reported clinical cases features the combination of an SRH and intraventricular hemorrhage (IVH), although Schievink et al. have described a patient with CSF xanthochromia and SRH, suggesting a combination of subarachnoid hemorrhage (SAH) and SRH. ${ }^{8}$ Nevertheless, despite extensive cases of idiopathic or angiographically negative $\mathrm{SAH}$ and $\mathrm{IVH},{ }^{2-7}$ the vascular or etiological source of SRH remains unexplained, and the relationship among SAH, IVH, and SRH remains unexamined.

Current clinical knowledge regarding patients with $\mathrm{SRH}$ is restricted to the aforementioned case report. We describe the first case series of patients with SRH.

\section{Methods}

We performed a retrospective analysis of all patients with diagnosed SRH at our institution over a 3-year period from January 2012 to January 2015. Collected data

ABBREVIATIONS IVH = intraventricular hemorrhage; MRA = MR angiography; $\mathrm{SAH}=$ subarachnoid hemorrhage; SRH = spontaneous retroclival hematoma. SUBMITTED October 21, 2014. ACCEPTED February 3, 2015.

INCLUDE WHEN CITING Published online August 7, 2015; DOI: 10.3171/2015.2.JNS142221.

DISCLOSURE The authors report no conflict of interest concerning the materials or methods used in this study or the findings specified in this paper. 
included clinical history, laboratory results, treatment, and review of all imaging studies (J.N.) performed.

\section{Illustrative Cases}

\section{Case 1}

A 58-year-old diabetic male experienced a bilateral frontal thunderclap headache while watching television. He had no recent trauma or injury, nor did he have a history of substance use. On examination, no focal neurological signs were found. Noncontrast CT demonstrated posterior fossa hemorrhage and IVH in a configuration typical of retroclival extraaxial blood (Fig. 1A). Magnetic resonance imaging confirmed the predominant subdural location of retroclival blood (Fig. 1B and C). Diagnostic digital subtraction angiograms obtained on Days 1 and 5 of admission revealed no source of the bleed and no evidence of vasospasm (Fig. 1D). Total spine MRI also revealed no abnormality (not shown). Transcranial Doppler ultrasound showed no evidence of vasospasm. The patient's hospital course included typical measures for intracranial pressure management and cerebral salt wasting followed by an uncomplicated discharge home. At the 30- and 90-day posthospitalization outpatient clinical follow-ups, the patient denied having any symptoms or complaints.

\section{Case 2}

A 64-year-old female experienced the sudden onset of a progressive worsening headache over the course of 1 day. She presented without focal neurological signs. Noncon-
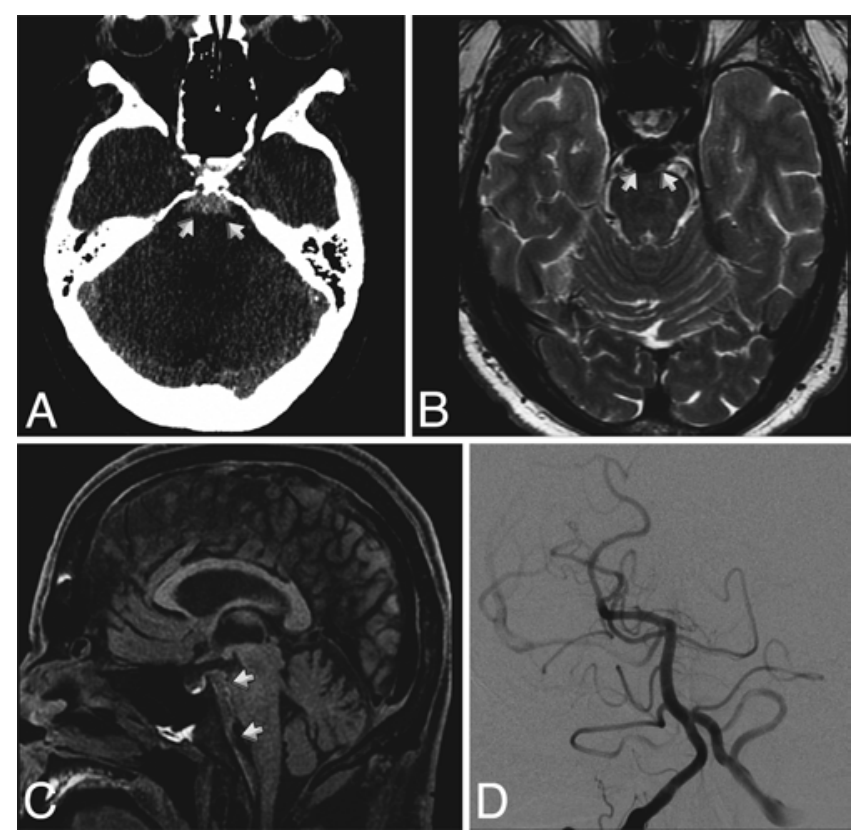

FIG. 1. A: Axial noncontrast CT demonstrates hemorrhage along the retroclival dura mater (arrows). B: Axial T2-weighted MR image demonstrates blood products conforming to the retroclival subdural space (arrows). C: Sagittal T1-weighted MR image shows to better advantage hemorrhagic collection along the retroclival dura (arrows). D: Digital subtraction angiogram, right vertebral artery injection, anteroposterior projection, is without vascular abnormality; a fetal left posterior cerebral artery is visible (normal variant). trast CT demonstrated a retroclival hematoma (Fig. 2A and B). Subsequently, MRI, MR angiography (MRA), and catheter angiography were performed, demonstrating no structural, tumoral, or angiographic cause of the hemorrhage (Fig. 2C and D). An uncomplicated 5-day hospital course with subsequent discharge followed. At the 90-day posthospitalization outpatient clinical follow-up, the patient denied having any symptoms or complaints.

\section{Case 3}

A 64-year-old man experienced the acute onset of severe occipital headache with subsequent diplopia. He had a history of hypertension. Admission CT scanning revealed SRH and IVH (Fig. 3A-C). Diagnostic digital subtraction angiograms on Day 2 of admission revealed no source of the bleeding and no evidence of vasospasm (Fig. 3D). Brain MRI, MRA, and spine MRI revealed no source of the bleed but did confirm the location of blood confined to the retroclival subdural space. The patient demonstrated no clinical or ultrasonographic signs of vasospasm during a week of hospitalization and was discharged. At the 90day and 1-year outpatient clinical follow-ups, the patient complained of occasional mild headaches without accompanying neurological signs or symptoms.

\section{Case 4}

A 67-year-old man developed acute-onset severe headache and neck pain. He became unresponsive in the emergency department. Admission CT scanning revealed SRH and punctate hemorrhage within the fourth ventricle (Fig. 4A and B). Subsequently, the patient underwent cerebral angiography, which showed no cause for the anatomical abnormality (Fig. 4C). On Day 2 of hospitalization, the patient underwent brain and total spine MRI (Fig. 4D-F),
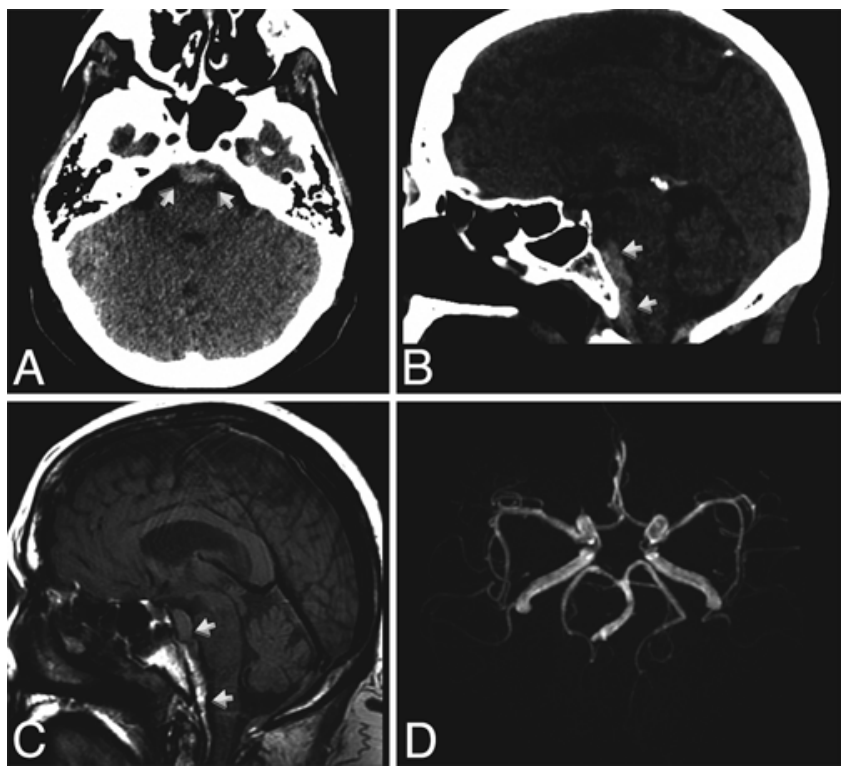

FIG. 2. A and B: Axial and sagittal noncontrast CT demonstrates hemorrhage along the retroclival dura (arrows). C: Sagittal T1-weighted MR image shows a layering hemorrhagic collection along the retroclival dura (arrows). D: Normal MR angiogram. 

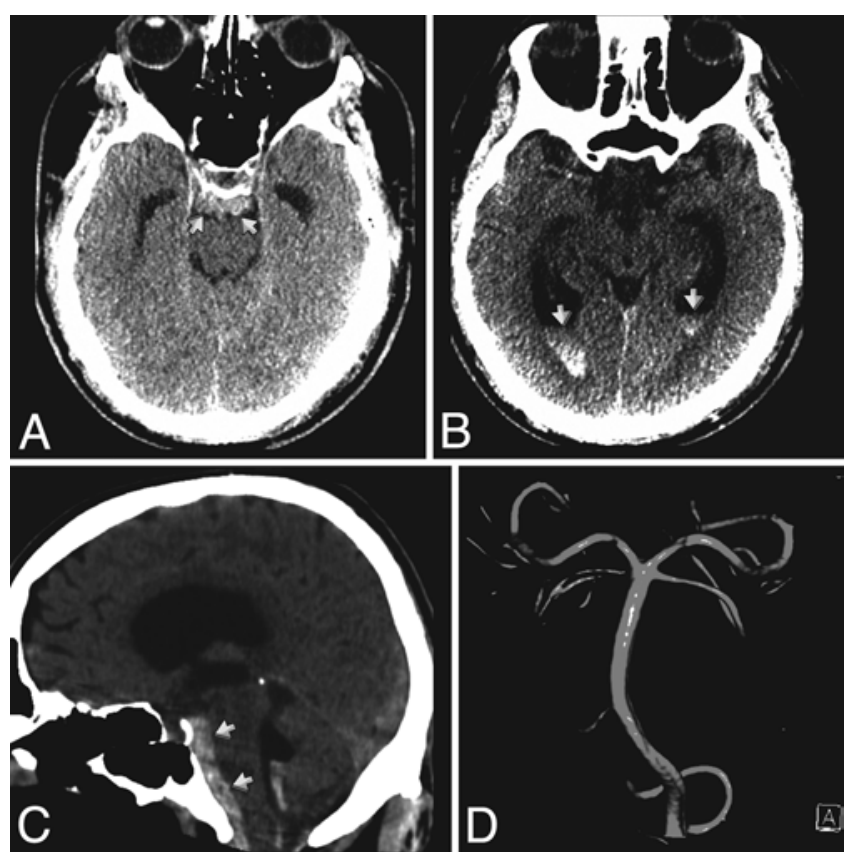

FIG. 3. Axial noncontrast CT demonstrates both SRH (arrows, A) and IVH (arrows, B). Sagittal noncontrast CT (C) demonstrates hemorrhagic collection conforming to the retroclival extraaxial space (arrows). Normal $3 \mathrm{D}$ left vertebral artery catheter angiogram (D).

which clarified the location of blood within the retroclival subdural space but did not reveal a vascular cause for the $\mathrm{SAH}$. At the 15-month follow-up, the patient described no neurological complaints.

\section{Discussion}

Each patient presented with a sudden-onset severe headache. None of them demonstrated sellar or pituitary abnormalities to suggest pituitary apoplexy. The differential diagnosis of thunderclap headache remains broad, including aneurysmal and nonaneurysmal causes. ${ }^{7}$ Schievink et al. have reported the only case within the extensive literature on angiographically negative CSF xanthochromia to include an SRH. ${ }^{8}$ With our cases, we add to this singular description by clarifying several points. First, 3 of the 4 patients did indeed have IVH in addition to the retroclival subdural hemorrhage. All patients were also appropriately evaluated for coagulopathic and/or traumatic etiologies of the hemorrhage, but no etiology was found. Moreover, all patients demonstrated a contained collection of retroclival blood that both clearly crossed the basioccipital synchondrosis and remained within a nondependent configuration along the retroclival dura.

One limitation of this study is the lack of pathological specimens to definitively evaluate the histo-anatomical location of retroclival blood in our patients. Some authors have argued for a more nuanced nomenclature when describing the meningeal coverings in this region given that the basilar plexus exists within the interdural (subdural) space between the 2-layered dura in this region and that a separately named layer, designated the "anterior pontine membrane" (free arachnoid membrane), separates the clival dura from the subarachnoid space. ${ }^{1}$ Although MRI, especially 3D high-resolution 3T MRI, helps identify retroclival blood within the extraaxial epidural or subdural
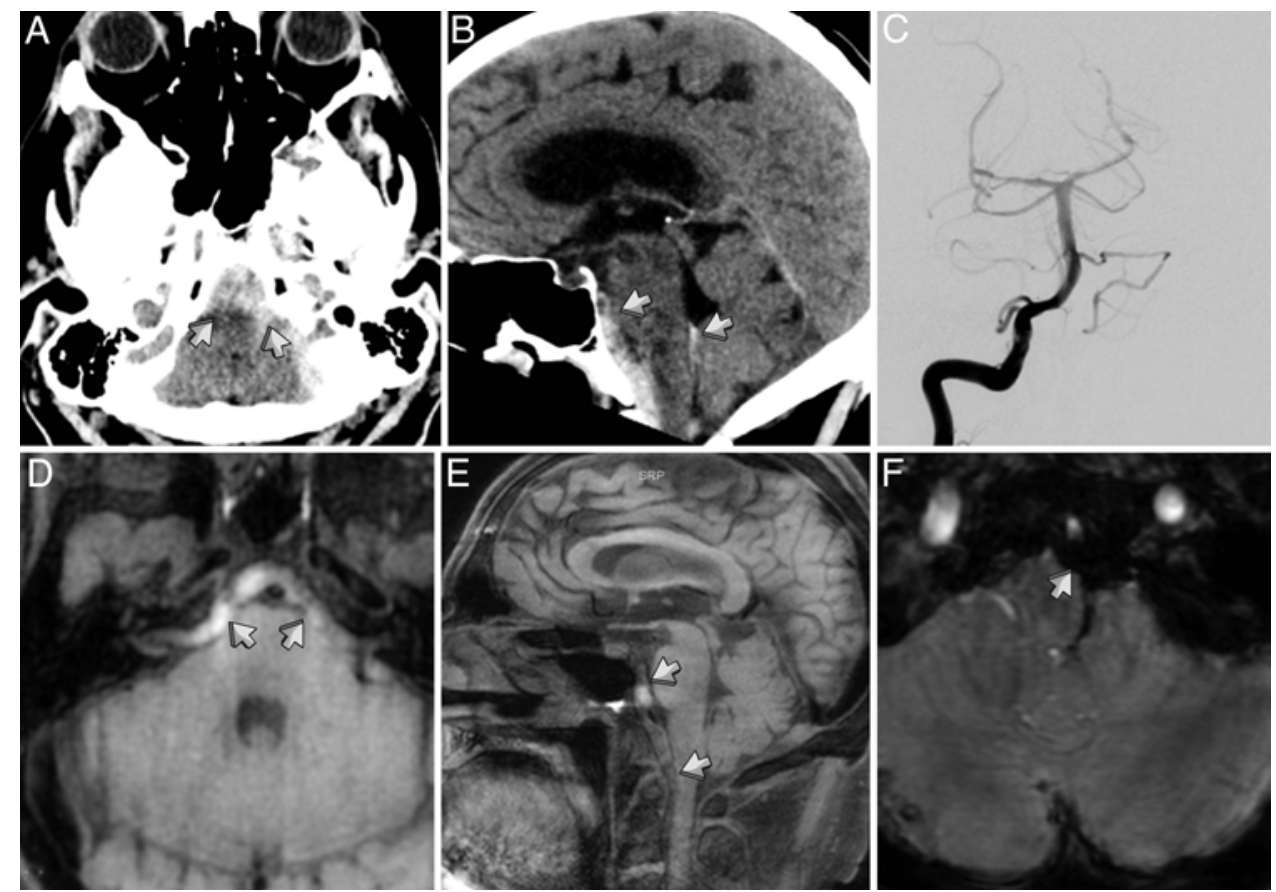

FIG. 4. A and B: Axial and sagittal noncontrast CT demonstrates hemorrhage along the retroclival dura as well as within the fourth ventricle. C: Normal catheter angiogram, right vertebral artery injection, anteroposterior projection. D and E: 3D CUBE T1-weighted MR images demonstrate retroclival subdural intrinsic T1 shortening caused by methemoglobin, which conforms to the retroclival extraaxial space (arrows). F: Susceptibility-weighted imaging confirms the presence of blood products in the retroclival subdural space (arrows). 
space rather than the subarachnoid space, no current imaging modality specifically allows evaluation of the dural border cell layer, which defines the meningeal dura.

Finally, although follow-up in our patients is limited to less than 2 years, none of the patients suffered the sequelae, namely vasospasm and delayed ischemia, typically associated with aneurysmal SAH. Just the opposite, patients had a remarkably benign hospitalization and convalescence, entirely consistent with the natural history of nonaneurysmal subarachnoid bleeds and prior literature on SRH.

\section{Conclusions}

Spontaneous retroclival hematoma, often associated with IVH, is a rare subtype of intracranial hemorrhage frequently recognized only when MRI demonstrates compartmentalization of the posterior fossa hemorrhage. Within the context of posterior fossa hemorrhage, catheter angiography remains the most important diagnostic investigation. Furthermore, when angiography fails to reveal an underlying lesion, SRH patients, like patients with traditional angiographically negative $\mathrm{SAH}$, enjoy a remarkably good prognosis.

\section{References}

1. Ayberk G, Ozveren MF, Aslan S, Yaman ME, Yaman O, Kayaci S, et al: Subarachnoid, subdural and interdural spaces at the clival region: an anatomical study. Turk Neurosurg 21:372-377, 2011

2. Boswell S, Thorell W, Gogela S, Lyden E, Surdell D: Angiogram-negative subarachnoid hemorrhage: outcomes data and review of the literature. J Stroke Cerebrovasc Dis 22:750-757, 2013

3. Dalyai R, Chalouhi N, Theofanis T, Jabbour PM, Dumont AS, Gonzalez LF, et al: Subarachnoid hemorrhage with negative initial catheter angiography: a review of 254 cases evaluating patient clinical outcome and efficacy of short- and long-term repeat angiography. Neurosurgery 72:646-652, 2013
4. Delgado Almandoz JE, Crandall BM, Fease JL, Scholz JM, Anderson RE, Kadkhodayan Y, et al: Diagnostic yield of catheter angiography in patients with subarachnoid hemorrhage and negative initial noninvasive neurovascular examinations. AJNR Am J Neuroradiol 34:833-839, 2013

5. Delgado Almandoz JE, Jagadeesan BD, Refai D, Moran CJ, Cross DT III, Chicoine MR, et al: Diagnostic yield of repeat catheter angiography in patients with catheter and computed tomography angiography negative subarachnoid hemorrhage. Neurosurgery 70:1135-1142, 2012

6. Lin N, Zenonos G, Kim AH, Nalbach SV, Du R, Frerichs $\mathrm{KU}$, et al: Angiogram-negative subarachnoid hemorrhage: relationship between bleeding pattern and clinical outcome. Neurocrit Care 16:389-398, 2012

7. Maslehaty H, Barth H, Petridis AK, Doukas A, Maximilian Mehdorn H: Special features of subarachnoid hemorrhage of unknown origin: a review of a series of 179 cases. Neurol Res 34:91-97, 2012

8. Schievink WI, Thompson RC, Loh CT, Maya MM: Spontaneous retroclival hematoma presenting as a thunderclap headache. Case report. J Neurosurg 95:522-524, 2001

9. Tomaras C, Horowitz BL, Harper RL: Spontaneous clivus hematoma: case report and literature review. Neurosurgery 37:123-124, 1995

10. Tubbs RS, Griessenauer CJ, Hankinson T, Rozzelle C, Wellons JC, Blount JP, et al: Retroclival epidural hematomas: a clinical series. Neurosurgery 67:404-407, 2010

11. van Rijn RR, Flach HZ, Tanghe HLJ: Spontaneous retroclival subdural hematoma. JBR-BTR 86:174-175, 2003

\section{Author Contributions}

Conception and design: Narvid. Acquisition of data: Narvid. Analysis and interpretation of data: Narvid. Drafting the article: Narvid. Reviewed submitted version of manuscript: all authors. Approved the final version of the manuscript on behalf of all authors: Narvid. Study supervision: Narvid.

\section{Correspondence}

Jared Narvid, Department of Neuroradiology, University of California, San Francisco, 505 Parnassus Ave., L-352, San Francisco, CA 94143-0628. email: jnarvid@ gmail.com. 